\title{
Z' Signature from Muon Pair Production at the LHC
}

\author{
J. Sá Borges \\ Universidade do Estado do Rio de Janeiro \\ E. Ramirez Barreto \\ Y. A. Coutinho \\ Universidade Federal do Rio de Janeiro \\ Rio de Janeiro, RJ, Brazil \\ E-mail: saborgesduerj.br, Elmerdif.ufrj.br, varadif.ufrj.br
}

\begin{abstract}
We search for signatures of the extra neutral gauge boson $Z^{\prime}$, predicted in some extensions of the Standard Model (SM), from the analysis of some distributions for $p+p \longrightarrow \mu^{+}+\mu^{-}+$ $X$, where the only exotic particle involved is $Z^{\prime}$. In addition to the invariant mass and charge asymmetry distributions, we propose in our search to use the transverse momentum distribution $p_{T}$ to distiguish the models. We do our calculation for $14 \mathrm{TeV}$ LHC center of mass energy, corresponding to $100 \mathrm{fb}^{-1}$ of luminosity, in order to compare our findings from some models with the distributions following from the Standard Model.
\end{abstract}

35th International Conference on High Energy Physics

July 22-28 2010

Paris, France 


\section{Models}

We consider three different approaches to physics beyond the SM: $E_{6}$ model in their low energy $\chi$ version [四, the left-right model based in the gauge group $S U(2)_{L} \times S U(2)_{R} \times U(1)_{B-L}$ and two versions (MIN [[]], RHN [四]) of the model with symmetry $S U(3)_{C} \times S U(3)_{L} \times U(1)_{X}$, called 3-3-1 model.

The model Lagrangian and couplings for the neutral current involving $Z$ and $Z^{\prime}$ is:

$$
\mathscr{L}^{N C}=-\frac{g}{2 \cos \theta_{W}} \sum_{f}\left[\bar{f} \gamma^{\mu}\left(g_{V}+g_{A} \gamma^{5}\right) f Z_{\mu}+\bar{f} \gamma^{\mu}\left(g_{V}^{\prime}+g_{A}^{\prime} \gamma^{5}\right) f Z_{\mu}^{\prime}\right]
$$

\begin{tabular}{||c|c|c|c|c||}
\hline \hline \multicolumn{2}{|c|}{$3-3-1$ MIN } & \multicolumn{2}{|c||}{$3-3-1$ RHN } \\
\hline$Z^{\prime} \bar{l} l$ & $-\frac{\sqrt{3}}{2} \sqrt{1-4 \sin ^{2} \theta_{W}}$ & $\frac{\sqrt{3}}{6} \sqrt{1-4 \sin ^{2} \theta_{W}}$ & $\frac{-1+4 \sin ^{2} \theta_{W}}{2 \sqrt{3-4 \sin ^{2} \theta_{W}}}$ & $-\frac{1}{2 \sqrt{3-4 \sin ^{2} \theta_{W}}}$ \\
\hline$Z^{\prime} \bar{u} u$ & $-\frac{1+4 \sin ^{2} \theta_{W}}{2 \sqrt{3-12 \sin ^{2} \theta_{W}}}$ & $\frac{1}{\sqrt{3-12 \sin ^{2} \theta_{W}}}$ & $\frac{3-8 \sin ^{2} \theta_{W}}{6 \sqrt{3-4 \sin ^{2} \theta_{W}}}$ & $-\frac{1}{2 \sqrt{3-4 \sin ^{2} \theta_{W}}}$ \\
\hline$Z^{\prime} \bar{d} d$ & $\frac{1-2 \sin ^{2} \theta_{W}}{2 \sqrt{3-12 \sin ^{2} \theta_{W}}}$ & $-\frac{1+2 \sin ^{2} \theta_{W}}{2 \sqrt{3-12 \sin ^{2} \theta_{W}}}$ & $\frac{3-2 \sin ^{2} \theta_{W}}{6 \sqrt{3-4 \sin ^{2} \theta_{W}}}$ & $-\frac{\sqrt{3-4 \sin ^{2} \theta_{W}}}{6}$ \\
\hline
\end{tabular}

\begin{tabular}{||c|c|c|c|c||}
\hline \hline \multicolumn{3}{|c|}{ Sym L-R } & \multicolumn{2}{c||}{$E_{6}-\chi$} \\
\hline$Z^{\prime} \bar{l} l$ & $\frac{-1+4 \sin ^{2} \theta_{W}}{2 \sqrt{\cos ^{2} \theta_{W}-\sin ^{2} \theta_{W}}}$ & $\frac{g_{A}^{\prime}}{\sqrt{\cos ^{2} \theta_{W}-\sin ^{2} \theta_{W}}}$ & $\frac{2 \sin \theta_{W}}{\sqrt{6}}$ & $\frac{\sin \theta_{W}}{\sqrt{6}}$ \\
\hline$Z^{\prime} \bar{u} u$ & $\frac{3-8 \sin ^{2} \theta_{W}}{6 \sqrt{\cos ^{2} \theta_{W}-\sin ^{2} \theta_{W}}}$ & $-\frac{\sqrt{\cos ^{2} \theta_{W}-\sin ^{2} \theta_{W}}}{2}$ & 0 & $\frac{\sin \theta_{W}}{\sqrt{6}}$ \\
\hline$Z^{\prime} \bar{d} d$ & $\frac{-3+4 \sin ^{2} \theta_{W}}{6 \sqrt{\cos ^{2} \theta_{W}-\sin ^{2} \theta_{W}}}$ & $\frac{\sqrt{\cos ^{2} \theta_{W}-\sin ^{2} \theta_{W}}}{2}$ & $-\frac{2 \sin \theta_{W}}{\sqrt{6}}$ & $-\frac{\sin \theta_{W}}{\sqrt{6}}$ \\
\hline
\end{tabular}
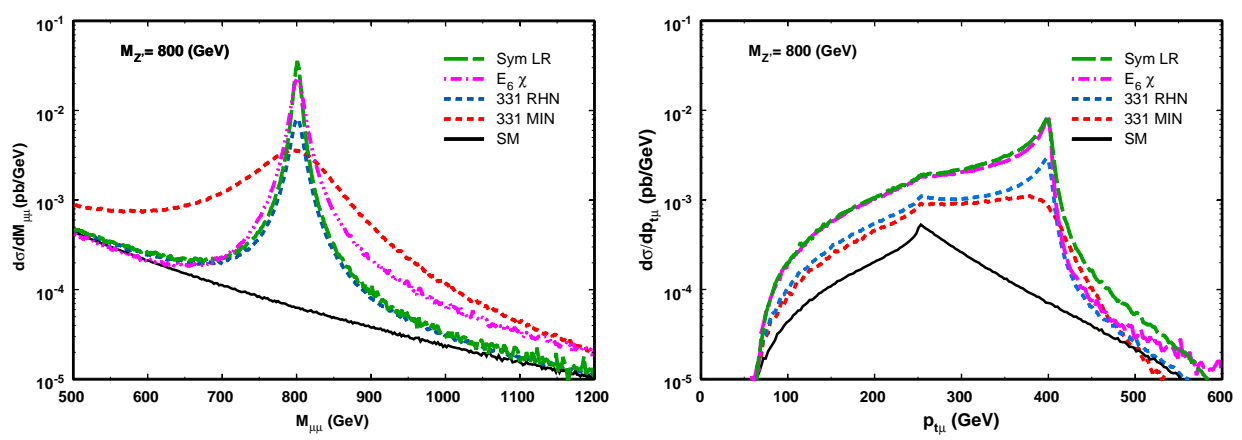


\section{Results}

We calculate the invariant mass, forward-backward asymmetry and transverse momentum distributions for $p+p \longrightarrow \mu^{+}+\mu^{-}+X$, at $14 \mathrm{TeV}$. All calculations of this work are performed with the CompHep package [四].

In the figure (left) we display the resulting muon pair mass distributions. One can observe that, for the 331-MIN, there are many $Z^{\prime}$ decay channels available and so the distribution is more flat than the others and also that, even for small dimuon mass values, the predicted number of dimons in 331-MIN, is larger than the SM one.

We study the angular distributions in order to analyze the forward-backward asymmetry $\left(\mathrm{A}_{F B}\right)$. This calculation is quite difficult because the direction of the interacting quarks are not determined. To face this difficulty one uses the kinematics of the dimuon system. As the elementary processes involve quarks with various momentum distributions, one can approximate the quark direction by the boost direction connecting the dimuon system with the beam axis.

As a consequence the assignment of quark direction can be obtained if one selects dimuon large rapidity events. The asymmetry calculated from all models, except the 331-MIN model (by $Z^{\prime}$ leptophobic character), is very sensitive to the $Z^{\prime}$ mass. We conclude that the $A_{F B}$ analysis can be used to disentangle the models.

In order to emphasize the role played by the exchange of an exotic neutral gauge boson, we have also calculated the fermion transverse momentum distribution. Two peaks are expected to appear in the muon $p_{T}$ distribution corresponding to one half of the resonance masses $\left(M_{Z}\right.$ and $\left.M_{Z^{\prime}}\right)$. We have applied a more strong cut in the dimuon mass $\left(M_{\mu \mu}>500 \mathrm{GeV}\right)$ and we present in the figure (right) our findings. We observe in this case that, for all models including the SM one, there is a peak at $250 \mathrm{GeV}$, which is related to the adopted invariant mass cut. The second peak keeps its position at $p_{T}=M_{Z^{\prime}} / 2$. Moreover, we observe a complete different shape for the distributions: the LRM exhibits a more pronounced peak while the $331-\mathrm{MIN}$ is flat due to its peculiar $Z^{\prime}$ width.

We claim that the mesure of forward-backward asymmetry and the muon $p_{T}$ distribution behavior can be used as a nice signature for $Z^{\prime}$ experimental search and for theoreticians to disentangle models.

\section{References}

[1] P. Langacker, et al. Phys. Rev. D 30 (1984) 1470. J. L. Hewett, et al. Phys. Rep. 183 (1989) 193.

[2] F. Pisano et al.,Phys. Rev. D 46 (1992) 410. P. H. Frampton, Phys. Rev. Lett. 69 (1992) 2889.

[3] J. C. Montero et al.,Phys. Rev. D 47 (1993) 2918. R. Foot et al., Phys. Rev. D 50 (1994) R34.

[4] A. Pukhov et al., Nucl. Instrum. Meth. A 534 (2004) 250. 